\title{
No association between the GSTM1 and GSTT1 polymorphism and endometrial cancer risk: A meta-analysis ${ }^{*}$
}

\author{
Marce-Amara Kpoghomou ${ }^{1 \#}$, Fatch W. Kalembo ${ }^{2}$, Joella Eldie Soatiana ${ }^{3}$ \\ ${ }^{1}$ Department of Epidemiology and Biostatistics, School of Public Health, Tong Ji Medical College, Huazhong University of Science \\ and Technology, Wuhan, China \\ ${ }^{2}$ Department of Maternal and Child Health, School of Public Health, Tong Ji Medical College, Huazhong University of Science and \\ Technology, Wuhan, China \\ ${ }^{3}$ Department of Ophthalmology, Tong Ji Medical College, Huazhong University of Science and Technology, Wuhan, China \\ Email: "kpogmarce@yahoo.fr
}

Received 13 April 2013; revised 15 May 2013; accepted 24 May 2013

Copyright (C) 2013 Marce-Amara Kpoghomou et al. This is an open access article distributed under the Creative Commons Attribution License, which permits unrestricted use, distribution, and reproduction in any medium, provided the original work is properly cited.

\begin{abstract}
Purpose: A number of case-control studies have been conducted to investigate the association of glutathione S-transferase (GST) genetic polymorphisms and endometrial carcinoma risk. However, these studies have yielded contradictory results. We therefore performed a meta-analysis to derive a more precise estimation of the association between polymorphisms on GSTM1, GSTT1 and endometrial carcinoma. Methods: Identification of relevant studies was carried out through a search in the following databases Medline, EMbase and China National Knowledge International (CNKI) up to March. 2013. All case-control studies that investigated the association between GSTM1 and GSTT1 gene polymorphisms and risk of endometrial cancer were included in the study. The pooled odds ratio (OR) was used for analyses of results and the corresponding $95 \%$ confidence intervals (CI) were estimated. Result: Six published case-control studies of association between the GSTM1 and GSTT1 polymorphism and endometrial cancer risk covering 3558 subjects were included in the metaanalysis, but the results indicated that the null genotypes of GSTM1 and GSTT1 polymorphisms were not associated with a significantly increased risk of endometrial cancer (for GSTM1: OR $=0.99$; $95 \% \mathrm{CI}$,
\end{abstract}

*Declaration of Conflicting Interests: The authors declared no potential conflicts of interests with respect to the research, authorship, and publication of this article.

Funding: The authors received no financial support for the research, authorship, and publication of this article.

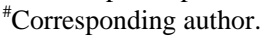

0.86 - 1.4; for GSTT1: OR = 0.96; 95\% CI, 0.80 - 1.14, respectively). Conclusion: This meta-analysis suggests that GSTM1 and GSTT1 polymorphism may not be associated with increased risk of endometrial cancer. To validate the association between polymorphism and endometrial cancer, further studies with larger numbers of participants worldwide are needed.

Keywords: GSTM1; GSTT1; Polymorphism; Endometrial Cancer; Meta-Analysis

\section{INTRODUCTION}

Endometrial cancer (EC) is the fourth most common cancer (after breast, lung, and colorectal cancer) in women in developed countries. Environmental factors have been associated with endometrial cancer in many studies, and a heritable component may predispose to the development of endometrial cancer [1]. In 2010, there were 43,470 newly diagnosed cases and 7950 deaths in the United States [2]. EC has predominantly been considered a genetic disease, characterized by sequential accumulation of genetic alterations [3,4]. Endometrial carcinogenesis is a multi-factorial interaction between environmental triggers and genetic susceptibility. Mutagens in the living environment can create DNA adducts and strand breaks, causing genomic instability. Loss of genomic stability and the resulting gene alterations appear to be a crucial molecular and pathogenic step that occurs early in the endometrial carcinogenesis process. Recent studies have revealed that genetic variants in genes controlling carcinogen metabolism, DNA repair and cell 
proliferation or apoptosis may be important in determining individual susceptibility to the occurrence and progression of EC [5,6]. Genetic polymorphisms in functionally critical genes have been suggested as risk factors for the development of a variety of cancers, including endometrial cancer [7]. Therefore, the identification of genetic factors may be helpful in better understanding the mechanisms underlying endometrial carcinogenesis and improving cancer detection.

Previous studies showed that the glutathione S-transferases (GSTs) genetic variant is related to human phase II detoxification enzymes. Cytosolic GSTs (GSTM and GSTT) play a role in the detoxification of the carcinogenic electrophiles of aflatoxin and polycyclic aromatic hydrocarbons (PAHs) in tobacco smoke. The mode of action of GSTs is considered to co-effect with activation and detoxification of tobacco carcinogens. Therefore, several studies found the association between the genetic polymorphisms of GSTs and the risk of cancer development [8-10]. GSTM1 facilitates the excretion of a wide range of carcinogens, reactive oxygen species and chemotherapeutic agents with a variety of substrate specificities [11]. The GSTT1 polymorphism is considered in the detoxification of environmental carcinogens, including 1,3 butadiene and ethylene oxide in tobacco smoke and ambient air [12]. The null GST (GST-null genotype) results in a completely loss of enzyme activity to bind with genotoxic substrates, including epoxides derived from aflatoxin and PAHs [13]. There are a large number of epidemiological studies that have been addressed to evaluate the association between GSTM1 and GSTT1 homozygous deletion polymorphisms and risk of endometrial cancer in diverse ethnicities [14-19]. However, this issue remains controversial because of inconsistent results among different studies. The possibilities for this discrepancy may be that some positive results might occur by chance and some negative findings might be caused by insufficient statistical power with small sample size. Additionally, different experimental design and selection bias should also be considered.

In order to provide strong evidence of the effects of GSTM1 and GSTT1 polymorphisms on endometrial cancer risk, we carried out a quantitative meta-analysis by combining data from all published case-control studies.

\section{MATERIALS AND METHODS}

\subsection{Selection Criteria and Search Strategy}

Identification of relevant studies was to carried out through a search of the following databases: Medline, EMbase and China National Knowledge International (CNKI) up to March 2013. Without language restrictions, using the following terms, including "endometrial can- cer”, “endometrial tumor”, “endometrial neoplasm”, “endometrial adenocarcinoma”, "glutathione S-transferase”, “GST”, “GSTM” and “GSTT”. All studies that examined the association/non association between GST gene polymorphisms and endometrial cancer were identified, 81 potentially relevant studies were searched. Of the 81 studies, 70 were irrelevant, while 5 studies were excluded because of various reasons (4 studies were conducted on GSTM1 and/or GSTT1 polymorphism and endometriosis, were not related to endometrial cancer and 1 study had insufficient genotype data for extraction). Finally, 6 studies met the inclusion criteria and were included in the meta-analysis (Figure 1).

The inclusion criteria were as following: case-control studies that investigated the association between GSTM1 and GSTT1 gene polymorphisms and risk of endometrial cancer with available full text; studies which presented original data and the number of null genotype of GSTM1 and GSTT1 in cases and controls. For each study, the following information were extracted: author, publication year, country of origin, number of cases and controls, number of null genotype for GSTM1 and GSTT1 in cases and controls and the adjusted and non-adjusted ORs of selected studies. Two authors independently assessed the articles for inclusion/exclusion, resolved disagreements, and reached consistency (Table 1).

\subsection{Statistical Analysis}

The association between GSTM1 and GSTT1 gene polymorphisms and cervical cancer was estimated by calculating pooled ORs and 95\% CIs. Odds ratio (OR) was used for analyses of results and their corresponding 95\% confidence intervals (CI) were estimated. The Cochran's Q-statistic was used to test heterogeneity, and the heterogeneity was considered statistically significant when $P \leq 0.1$ [20]. We also quantified the effect of heterogenity by the $I^{2}$ test. $I^{2}$ ranges between $0 \%$ and $100 \%$ and represents the proportion of inter-study variability that can be attributed to heterogeneity rather than chance. $I^{2}$ values of $25 \%$, $50 \%$ and $75 \%$ were defined as low, moderate and high estimates, respectively. When a significant $Q$ test $(P<0.10$ or $>50 \%)$ indicated heterogenity across studies, the random effects model was used for meta-analysis; otherwise, the fixed effects model was used [21]. Potential publication bias was estimated by constructing funnel plots [22]. As asymmetric funnel plot indicated a relationship between effect and study size, which suggested the possibility of either publication bias or a systematic difference between smaller and larger studies (small study effects). Furthermore, publication bias was assessed by Egger's test [23]. Studies were categorized into subgroups based on ethnicity and smoking status. The data analysis was performed (STATA, version 10). 


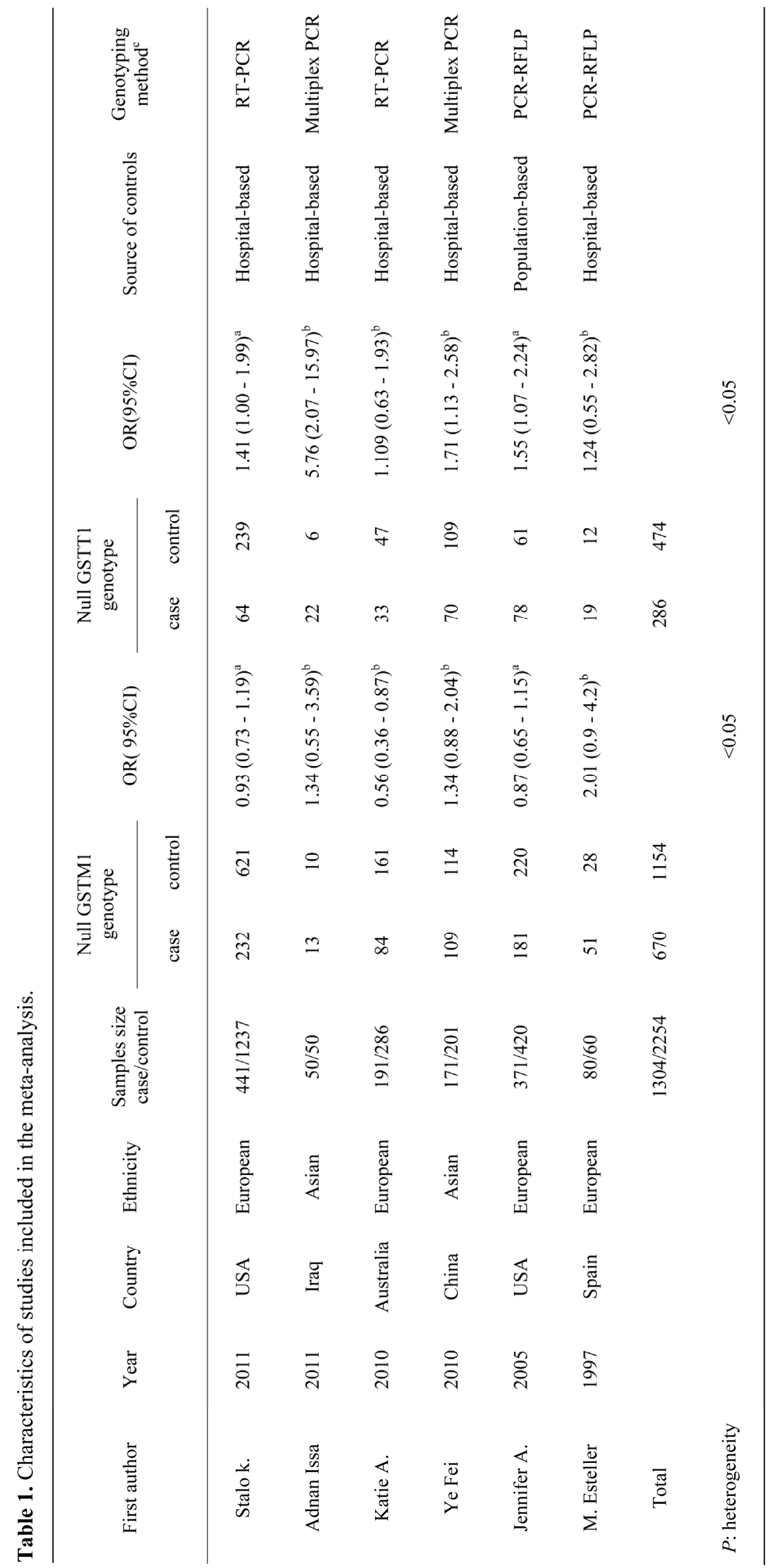




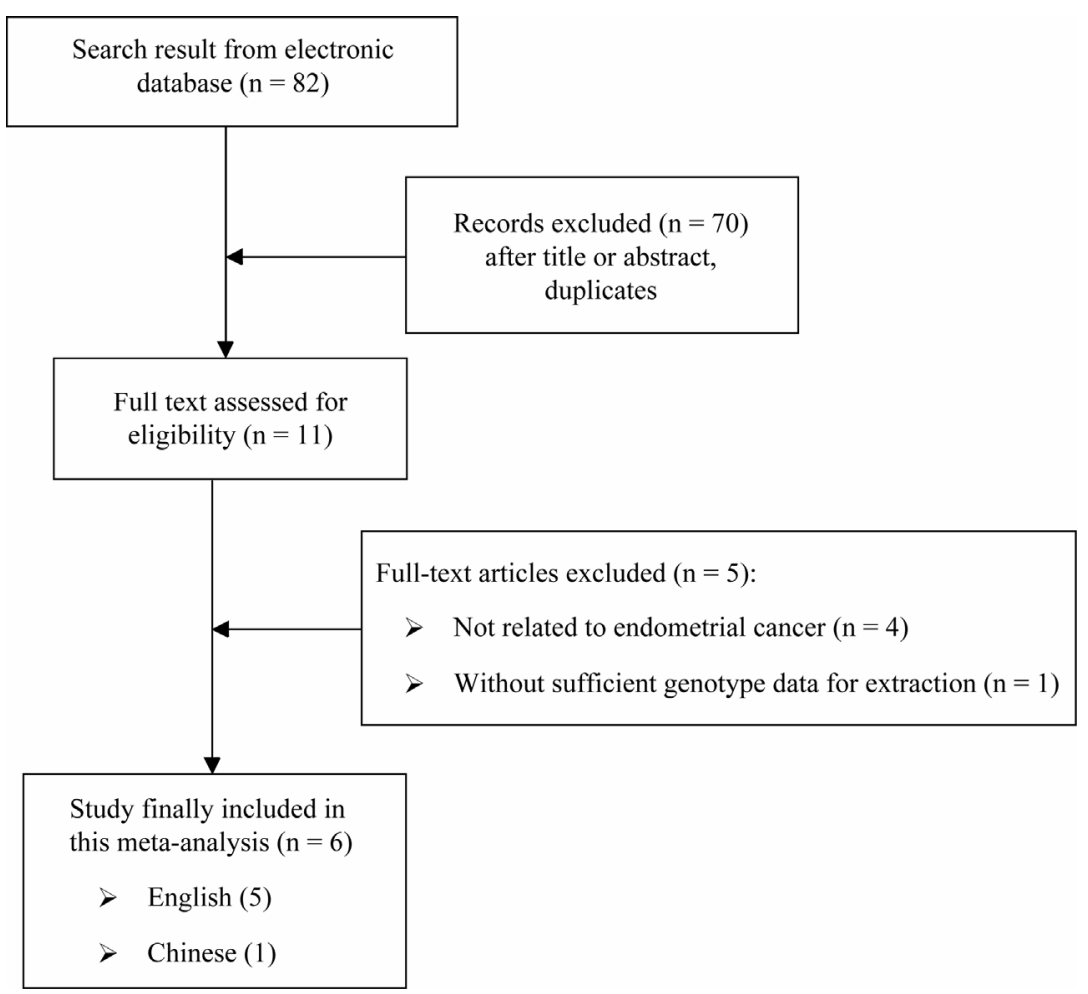

Figure 1. Flow chart showing study selection procedure.

\section{RESULTS}

\subsection{Eligible Studies and Meta-Analysis Databases}

We identified 81 articles through the initial computerized search of published work. After reading titles, abstracts, 11 articles were retained. For the analysis of GSTM1 or GSTT1, after discarding 4 articles [24-27] because they were not related to endometrial cancer and 1 article [5] due to insufficient genotype data, 6 case control studies [14-19] finally met our criteria for inclusion. Among them, 5 studies were published in English and 1 study in Chinese. For the analyses stratified by smoking, three studies $[14,17,18]$ for GSTM1 and GSTT1 were included.

\subsection{Heterogeneity Result}

Cochran's $Q$ tests indicated heterogeneity exists in different studies in the analysis. Significant heterogeneity across studies was present in overall analyses (for GSTM1: $I^{2}=65.8 \%$ and for GSTT1: $I^{2}=82.9 \%$ ) and subgroup analyses by ethnicity $\left(I^{2}=74.2 \%\right.$, for GSTM1 and $I^{2}=74 \%$ for GSTT1).

\subsection{Meta-Analysis Results}

A total of 6 case-control studies were included in the meta-analysis of GSTM1 and GSTT1 (1304 cases and

\section{4 controls) (Table 1).}

For GSTM1 polymorphism, the overall meta-analysis showed no statistically significant association between endometrial cancer risk and GSTM1 null genotype (OR $=0.99,95 \%$ CI: $0.86-1.4, P=0.86$ ) (Figure 2(a)). In subgroup analysis, no significant association was found in European or in Asian (Figure 3(a)).

For GSTT1 polymorphism, null genotype was not also associated with an increased risk of Endometrial cancer (OR = 0.96, 95\% CI: 0.80 - 1.14, $P=0.61$ ) (Figure 2(b)). Analysis stratified by ethnicity indicated that GSTT1 null genotype was not significantly associated with endometrial cancer risk in Europeans or Asians (Figure 3(b)).

\subsection{Sensitivity}

In sensitivity analysis by temporarily excluding individual studies, no single study substantially affected the pooled OR, indicating that the results of these meta analyses were stable.

\subsection{Publication Bias}

For analysis of publication bias, Begg's funnel plot were generated to assess potential publication bias for GSTM1 and GSTT1 (Figures 4(a) and (b)), and the symmetry of the funnel plot showed no evidence of publication bias. Also the $P$ values of the Egger's test for GSTM1 and GSTT1 were 0.40 and 0.38 , respectively. 
Study

$\%$

ID

OR $(95 \% \mathrm{CI}) \quad$ Weight

Stalo Karageorgi (2011)

Adnan Issa A1-Badran (2011)

Katie A. Ashton (2010)

Ye Fei (2010)

Jennifer A. Doherty (2005)

M Esteller (1997)

Overall $(\mathrm{I}$-squared $=65.8 \%, \mathrm{p}=0.012)$

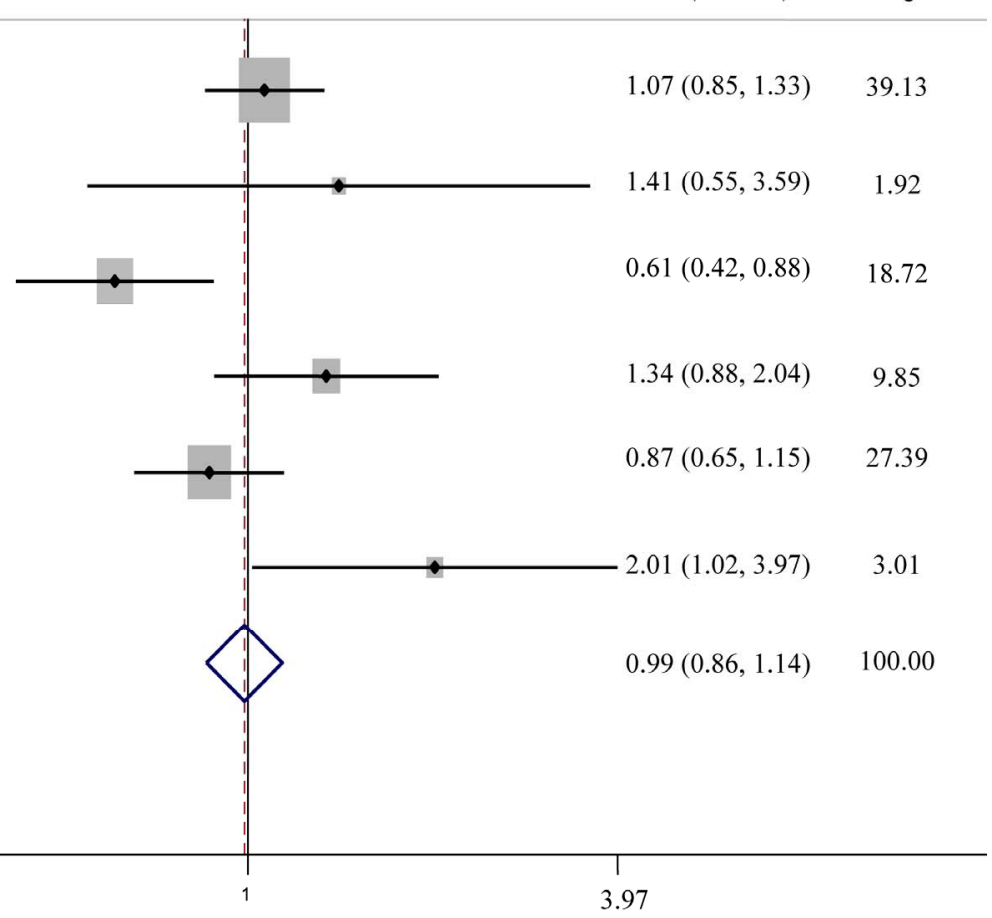

(a)

Study

ID

OR $(95 \%$ CI $) \quad$ Weight

Stalo Karageorgi (2011)

Adnan Issa Al-Badran (2011)

Katie A. Ashton (2010)

Ye Fei (2010)

Jennifer A. Doherty (2005)

M Esteller (1997)

Overall $(\mathrm{I}$-squared $=82.9 \%, \mathrm{p}=0.000)$

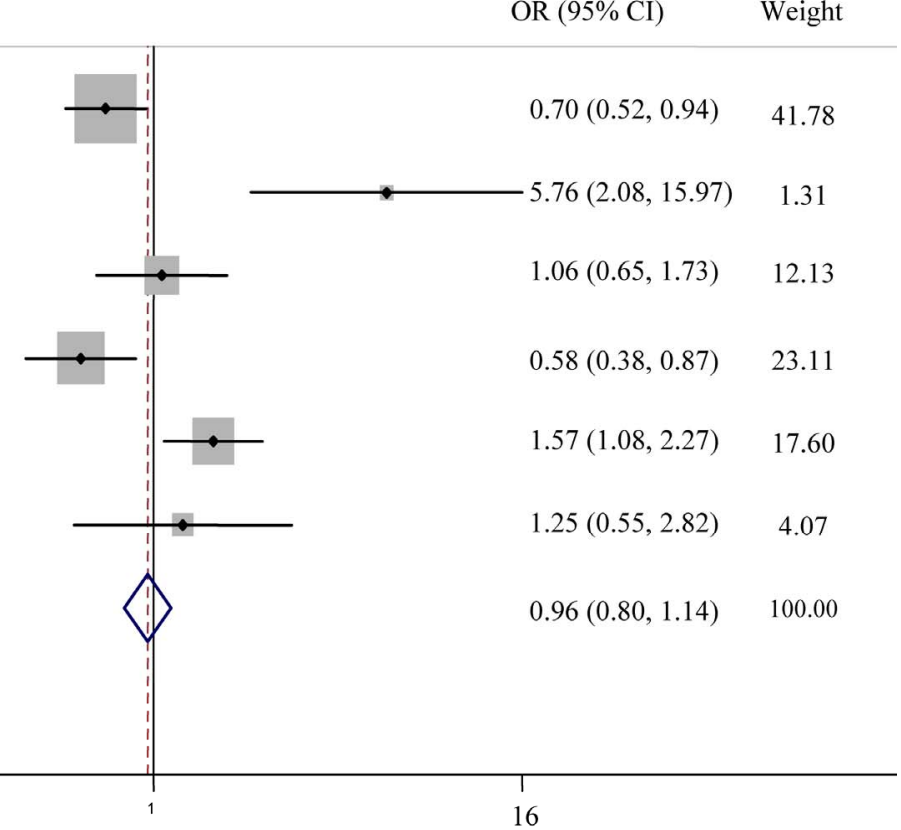

(b)

Figure 2. (a) Forest plot (random-effects model) of meta analysis regarding association of GSTM1 status and prostate cancer risk. The squares and horizontal lines correspond to the study-specific OR and 95\% CI. The area of the squares reflects the weight (inverse of the variance). The diamonds represent the summary OR and 95\% CI; (b) Forest plot (random-effects model) of Meta analysis regarding association of GSTT1 status and Prostate cancer risk. The squares and horizontal lines correspond to the study-specific OR and 95\% CI. The area of the squares reflects the weight (inverse of the variance). The diamonds represent the summary OR and 95\% CI. 
Study

$\%$

ID

OR $(95 \% \mathrm{CI})$

Weight

european

Stalo Karageorgi (2011)

Katie A. Ashton (2010)

Jennifer $\Lambda$. Doherty (2005)

M Esteller (1997)

Subtotal $($ I-squared $=74.2 \%, p=0.009)$

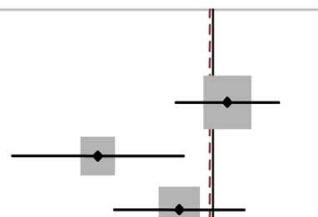

$1.07(0.85,1.33)$

39.13

$0.61(0.42,0.88)$

18.72

$0.87(0.65,1.15) \quad 27.39$

$2.01(1.02,3.97) \quad 3.01$

Asian

Adnan Issa Al-Badran (2011)

Ye Fei (2010)

Subtotal $(\mathrm{I}$-squared $=0.0 \%, \mathrm{p}=0.929)$

Overall (I-squared $=65.8 \%, p=0.012)$

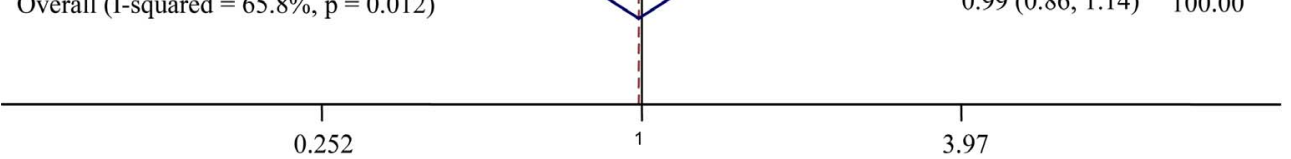

(a)

Study

ID

OR $(95 \% \mathrm{CI}) \quad$ Weight

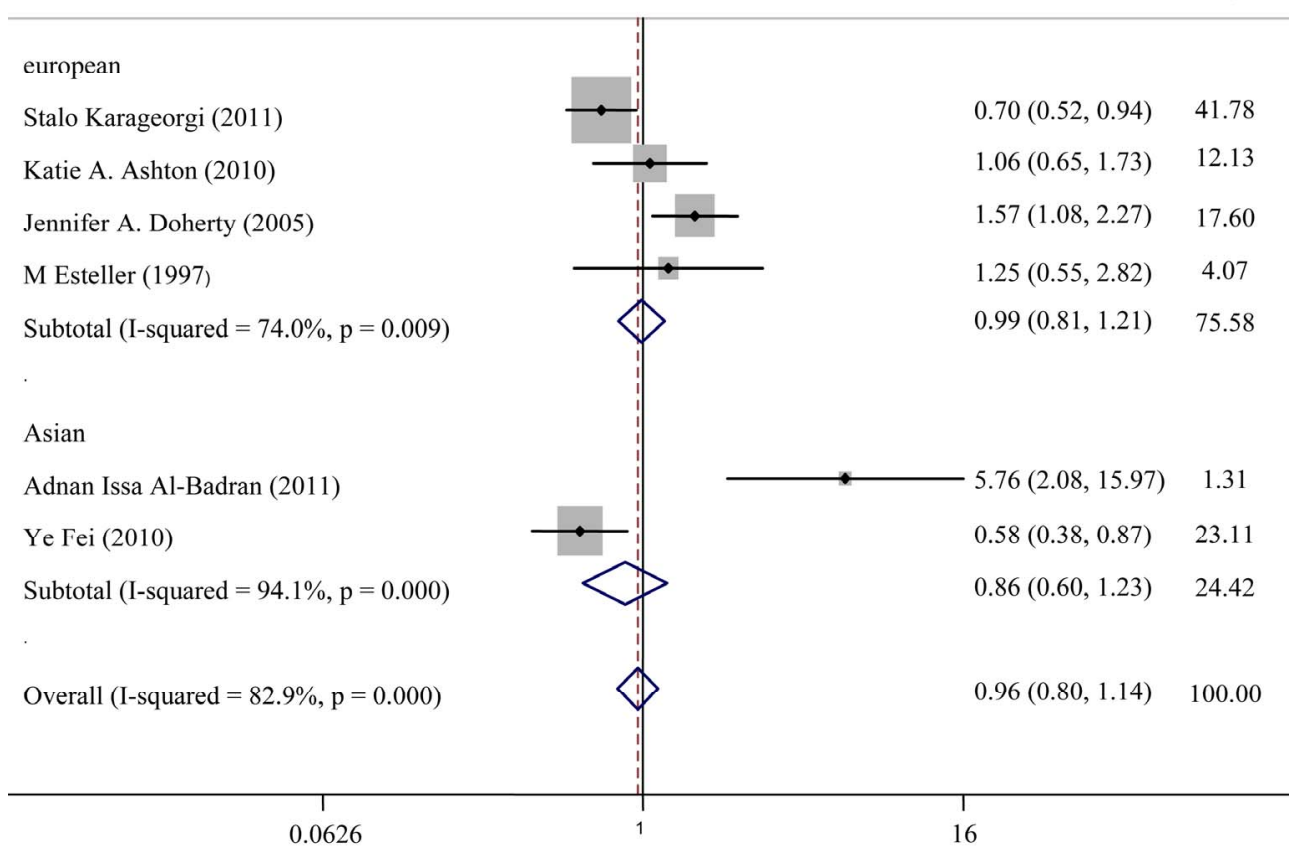

(b)

Figure 3. (a) Forest plot (random-effects model) of meta analysis regarding association of GSTM1 status and ethnicity. The squares and horizontal lines correspond to the study-specific OR and 95\% CI. The area of the squares reflects the weight (inverse of the variance). The diamonds represent the summary OR and 95\% CI; (b) Forest plot (random-effects model) of Meta analysis regarding association of GSTT1 status and ethnicity. The squares and horizontal lines correspond to the study-specific OR and 95\% CI. The area of the squares reflects the weight (inverse of the variance). The diamonds represent the summary OR and 95\% CI. 


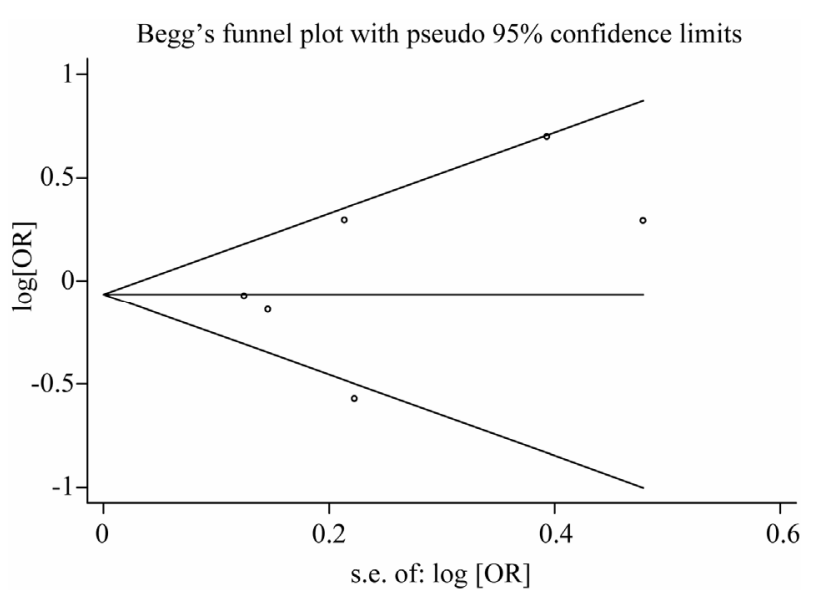

(a)

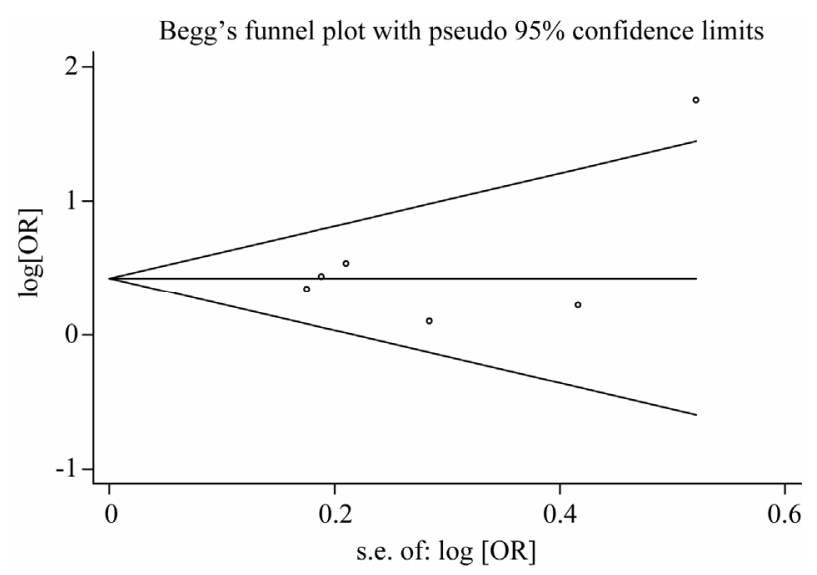

(b)

Figure 4. (a) Begg's funnel plot for publication bias test [for Null versus Present], for GSTM1 Gene Polymorphism and Endometrial Cancer Risk. Each point represents a separate study for the indicated association. Log [or], natural logarithm of OR. Horizontal line, mean effect size; (b) Begg's funnel plot for publication bias test [for Null versus Present], for GSTT1 Gene Polymorphism and Endometrial Cancer Risk. Each point represents a separate study for the indicated association. Log [or], natural logarithm of OR. Horizontal line, mean effect size.

\section{DISCUSSION}

The glutathiones S-transferases (GSTs) are the most important parts of phase II superfamily of metabolism enzymes. In humans, there are several GST classes that were encoded by distinct gene family [28]. Among them, GSTM1and GSTT1 should be pointed out because a polymorphic deletion of these genes may influence the enzyme activity, and eventually increase vulnerability to genotoxic damage [29,30]. Based on these backgrounds, the association has been intensively investigated between GSTM1and GSTT1 polymorphisms and risk of endometrial cancer [14-19]. Unfortunately, most of the studies had only a few hundred of participants, even less, which is too small to evaluate the overall effects precisely.
Meta-analysis has been considered to be a powerful tool to overcome this problem by combining the results from independent studies together. In this meta-analysis, we found that the null genotypes of GSTM1 and GSTT1 polymorphisms were not associated with a significantly increased risk of endometrial cancer, suggesting that GSTM1 and GSTT1 polymorphisms may not be involved in the development of endometrial cancer. Notably, the between-study heterogeneity was observed in both overall analyses and some subgroup analyses, further studies therefore are warranted to confirm these findings.

After subgroup analysis according to ethnicity, not significantly increased risks were observed in European population and in Asian. The possibilities of the conflicting results among diverse ethnicities may be that the GSTM1and GSTT1 polymorphisms have different effects on the risk of endometrial cancer in different genetic backgrounds and environment which are they exposed to. The frequency of GSTM1and GSTT1 null genotype in European and Asian were 88.24\%, $11.76 \%$ and $75.58 \%$, $24.42 \%$ respectively, which indicated ethnic differences. Additionally, the small sample size should also be taken into consideration because limited sample size may not have enough statistical power to detect a real effect or generate a fluctuated estimation.

To investigate potential gene-environment interaction, the lack of GST activities caused by an inherited deletion of the GST has been reported to increase the risk of several tobacco-related cancers [31-34]. In contrast, the studies included in this meta-analysis showed no significant association of endometrial cancer in GSTM1 and GSTT1 polymorphism $[14,17,18]$. It was therefore hypothesized that smoking and GST genotype may not synergistically influence the endometrial cancer development, which provides strong evidences for the lack of association between GSTs and endometrial cancer risk.

As the publication of findings often depends on the expectation of researchers, false-negative results may be suppressed or false-positive results magnified [35,36]. The results of this study, however, did not show any significant publication bias.

Although our result of this meta-analysis is constructive, its limitations and some potential bias should be addressed. First, despite that a well-designed search strategy was used to identify eligible studies, it was possible that some relevant studies were not included. This study only focused on full-text papers published in English and Chinese, so some eligible studies in other languages or in other databases might be missed. Secondly, adjustments over age, gender and other environmental factors such as drinking of alcohol might help to detect the association between GSTM1, GSTT1 and endometrial cancer. If available detailed individual data are enough for an adjusted estimate in the future, a more precise analysis 
should be conducted. Thirdly, ethnicity was determined roughly by the subject's country due to inadequate availability of data, and this classification can help us have a regional concept of functions of these genes. In addition, the sample size was relatively small in the meta-analysis, especially in subgroup analysis. Finally because all the studies were designed with retrospective studies, we cannot clearly determine the causal relationship between the risk factor and endometrial cancer. Given the limitations and biases above, the conclusions or interpretations made from the results of this Meta analysis should be explained with caution.

\section{CONCLUSION}

This meta-analysis involving 6 studies and 3558 subjects suggests that GSTM1 and GSTT1 polymorphism may not be associated with the risk of endometrial cancer among Europeans and Asians. Future well designed large studies might be necessary to validate this association in different populations, taking into account environmental factors in the susceptibility to endometrial cancer.

\section{REFERENCES}

[1] Gayther, S.A. and Pharoah, P.D. (2010) The inherited genetics of ovarian and endometrial cancer. Current Opinion in Genetics \& Development, 20, 231-238. doi:10.1016/j.gde.2010.03.001

[2] Jemal, A., et al. (2010) Cancer statistics, 2010. CA: A Cancer Journal for Clinicians, 60, 277-300. doi:10.3322/caac. 20073

[3] Lax, S.F. (2004) Molecular genetic pathways in various types of endometrial carcinoma: From a phenotypical to a molecular-based classification. Virchows Archiv, 444, 213223. doi:10.1007/s00428-003-0947-3

[4] Soliman, P.T., et al. (2005) Risk factors for young premenopausal women with endometrial cancer. Obstetrics \& Gynecology, 105, 575-580. doi:10.1097/01.AOG.0000154151.14516.f7

[5] Yang, H.P., et al. (2010) Common genetic variation in the sex hormone metabolic pathway and endometrial cancer risk: Pathway-based evaluation of candidate genes. Carcinogenesis, 31, 827-833. doi:10.1093/carcin/bgp328

[6] Zucchetto, A., et al. (2009) Hormone-related factors and gynecological conditions in relation to endometrial cancer risk. European Journal of Cancer Prevention, 18, 316-321. doi:10.1097/CEJ.0b013e328329d830

[7] Meyer, L.A., et al. (2008) Genetic polymorphisms and endometrial cancer risk. Expert Review of Anticancer Therapy, 8, 1159-1167. doi:10.1586/14737140.8.7.1159

[8] Carlsten, C., et al. (2008) Glutathione S-transferase M1 (GSTM1) polymorphisms and lung cancer: A literaturebased systematic HuGE review and meta-analysis. American Journal of Epidemiology, 167, 759-774. doi:10.1093/aje/kwm383
[9] Mo, Z., et al. (2009) An updating meta-analysis of the GSTM1, GSTT1, and GSTP1 polymorphisms and prostate cancer: A HuGE review. Prostate, 69, 662-688. doi:10.1002/pros.20907

[10] Zhuo, X., et al. (2009) GSTM1 and GSTT1 polymorphisms and nasopharyngeal cancer risk: An evidencebased meta-analysis. Journal of Experimental \& Clinical Cancer Research, 28, 46. doi:10.1186/1756-9966-28-46

[11] Rebbeck, T.R. (1997) Molecular epidemiology of the human glutathione S-transferase genotypes GSTM1 and GSTT1 in cancer susceptibility. Cancer Epidemiology, Biomarkers \& Prevention, 6, 733-743. doi:10.1016/S1383-5742(00)00050-8

[12] Landi, S. (2000) Mammalian class theta GST and differential susceptibility to carcinogens: A review. Mutation Research, 463, 247-283.

[13] Hayes, J.D. and Pulford, D.J. (1995) The glutathione Stransferase supergene family: Regulation of GST and the contribution of the isoenzymes to cancer chemoprotection and drug resistance. Critical Reviews in Biochemistry and Molecular Biology, 30, 445-600. doi:10.3109/10409239509083491

[14] Karageorgi, S., et al. (2011) GSTM1 and GSTT1 copy number variation in population-based studies of endometrial cancer risk. Cancer Epidemiology, Biomarkers \& Prevention, 20, 1447-1452. doi:10.1158/1055-9965.EPI-11-0190

[15] Al-Badran, A.I. (2011) Genetic polymorphisms of GSTM1 and GSTT1 genes and endometrial cancer in Basrah, South of Iraq. Journal of Basrah Researches (Sciences), 37, 54-59.

[16] Fei, Y., et al. (2010) Correlation of GSTM1 and GSTT1 genetic polymorphism with the susceptibility in endometriai cancer. Chinese Journal of Practical Gynecology and Obstetrics, $\mathbf{8}$.

[17] Ashton, K.A., et al. (2010) Polymorphisms in genes of the steroid hormone biosynthesis and metabolism pathways and endometrial cancer risk. Cancer Epidemiology, 34, 328-337. doi:10.1016/j.canep.2010.03.005

[18] Doherty, J.A., et al. (2005) Genetic factors in catechol estrogen metabolism in relation to the risk of endometrial cancer. Cancer Epidemiology, Biomarkers \& Prevention, 14, 357-366. doi:10.1158/1055-9965.EPI-04-0479

[19] Esteller, M., et al. (1997) Susceptibility to endometrial cancer: Influence of allelism at p53, glutathione S-transferase (GSTM1 and GSTT1) and cytochrome P-450 (CYP1A1) loci. British Journal of Cancer, 75, 1385-1388. doi:10.1038/bjc.1997.235

[20] Zintzaras, E. and Ioannidis, J.P. (2005) HEGESMA: Genome search meta-analysis and heterogeneity testing. Bioinformatics, 21, 3672-3673. doi:10.1093/bioinformatics/bti536

[21] Viechtbauer, W. (2007) Confidence intervals for the amount of heterogeneity in meta-analysis. Statistics in Medicine, 26, 37-52. doi:10.1002/sim.2514

[22] Begg, C.B. and Mazumdar, M. (1994) Operating characteristics of a rank correlation test for publication bias. Biometrics, 50, 1088-1101. doi:10.2307/2533446 
[23] Egger, M., et al. (1997) Bias in meta-analysis detected by a simple, graphical test. British Medical Journal, 315, 629-634. doi:10.1136/bmj.315.7109.629

[24] Arvanitis, D.A., et al. (2003) CYP1A1, CYP19, and GSTM1 polymorphisms increase the risk of endometriosis. Fertility and Sterility, 79, 702-709. doi:10.1016/S0015-0282(02)04817-3

[25] Swiersz, L.M. (2002) Role of endometriosis in cancer and tumor development. Annals of the New York Academy of Sciences, 955, 281-295, 396-406.

[26] Baxter, S.W., Thomas, E.J. and Campbell, I.G. (2001) GSTM1 null polymorphism and susceptibility to endometriosis and ovarian cancer. Carcinogenesis, 22, 63-65. doi:10.1093/carcin/22.1.63

[27] Arvanitis, D.A., et al. (2001) Low-penetrance genes are associated with increased susceptibility to endometriosis. Fertility and Sterility, 76, 1202-1206. doi:10.1016/S0015-0282(01)02865-5

[28] Strange, R.C., et al. (2001) Glutathione-S-transferase family of enzymes. Mutation Research, 482, 21-26. doi:10.1016/S0027-5107(01)00206-8

[29] Norppa, H. (2004) Cytogenetic biomarkers and genetic polymorphisms. Toxicology Letters, 149, 309-334. doi:10.1016/j.toxlet.2003.12.042

[30] Hayes, J.D. and Strange, R.C. (2000) Glutathione S-trans- ferase polymorphisms and their biological consequences. Pharmacology, 61, 154-166. doi:10.1159/000028396

[31] Kumar, V., et al. (2011) Association of GSTM1 and GSTT1 polymorphism with lipid peroxidation in benign prostate hyperplasia and prostate cancer: A pilot study. Disease Markers, 30, 163-169.

[32] Van Emburgh, B.O., et al. (2008) Polymorphisms in CYP1B1, GSTM1, GSTT1 and GSTP1, and susceptibility to breast cancer. Oncology Reports, 19, 1311-1321.

[33] van der Hel, O.L., et al. (2003) No modifying effect of NAT1, GSTM1, and GSTT1 on the relation between smoking and colorectal cancer risk. Cancer Epidemiology, Biomarkers \& Prevention, 12, 681-682.

[34] Kietthubthew, S., Sriplung, H. and Au, W.W. (2001) Genetic and environmental interactions on oral cancer in Southern Thailand. Environmental and Molecular Mutagenesis, 37, 111-116. doi:10.1002/em.1018

[35] Zhang, D., et al. (2008) A meta-analysis of DNA repair gene XPC polymorphisms and cancer risk. Journal of Human Genetics, 53, 18-33. doi:10.1007/s10038-007-0215-5

[36] Salanti, G., Sanderson, S. and Higgins, J.P. (2005) Obstacles and opportunities in meta-analysis of genetic association studies. Genetics in Medicine, 7, 13-20. doi:10.1097/01.GIM.0000151839.12032.1A 